TAO, Vol. 12, No. 3, 503-524, September 2001

\title{
Linear and Nonlinear Behaviors of Soft Soil Layers Using Lotung Downhole Array in Taiwan
}

\author{
Huey-Chu Huang ${ }^{1, *}$, Chie-Song Shieh ${ }^{1}$, and Hung-Chie Chiu ${ }^{2}$ \\ (Manuscript received 5 February 2001, in final form 18 July 2001)
}

\begin{abstract}
We analyze the acceleration records of a vertical array to study the linear and nonlinear behavior of the soft soil layers at LSST site, Lotung, Taiwan. This array includes five triaxial accelerometers deployed at depths of $0,6,11,17$ and $47 \mathrm{~m}$. During a 6-year operation from 1985 to 1990,30 earthquakes $\left(4.0 \leq M_{L} \leq 6.5\right)$ triggered this array. The maximum PGA value is 258 gals recorded at the surface station. Spectral analyses show that the strong motion (PGA $>150$ gals) causes the peaks of ratios to shift to lower predominant frequencies. The averaged spectral ratios of 15 well-recorded weak motion data (PGA $<100$ gals) are selected as a reference; the shift of the maximum predominant frequency can reach $20 \%$. Compared with the weak motions, the strong motions also decrease the amplification factor. The maximum reduction of the amplification can reach $50 \%$. The results of waveform simulation show that the linear model based on the Haskell method can well predict the weak motions at various depths. However, this linear model does not work for the strong motion data. Here a nonlinear numerical scheme, such as DESRA-2, is required and can significantly improve the simulation results although the PGA value at the surface station is still underestimated. Overall, the nonlinear numerical calculation is feasible to predict the strong motions for a horizontal layered structure.
\end{abstract}

(Key words: PGA, Spectral ratio, Predominant frequency, Amplification factor)

\section{INTRODUCTION}

Linear and nonlinear site effects have been examined in several studies recently (e.g., Joyner and Chen 1975; Yu et al. 1993; Aguirre and Irikura 1995, 1997). Basically, the ampli-

\footnotetext{
${ }^{1}$ Institute of Applied Geophysics, National Chung Cheng University, Ming-Hsiung, Chia-Yi, Taiwan, ROC

${ }^{2}$ Institute of Earth Sciences, Academia Sinica, Nankang, Taipei, Taiwan, R.O.C

* Corresponding author address: Prof. Huey-Chu Huang, Institute of Applied Geophysics, National Chung Cheng University, No.160 San-Hsing, Ming-Hsiung, Chia-Yi, 621, Taiwan, ROC

E-mail: seihuey@eq.ccu.edu.tw
} 
fication of the seismic waves originates from the strong contrast between the physical properties of the rocks and the sediments. To evaluate this amplification, the seismic response of the soil is treated as a linear behavior under low levels of strain. But for larger stress-strain levels, the results of laboratory testing of soil samples showed a nonlinear relation that represents the nonlinear character of the soil response. Some authors have been trying to find the observational evidences of the nonlinearity from seismological data and estimate how much it influences strong ground motions (Chin and Aki 1991; Beresnev et al. 1995a; Beresnev et al. 1998a, b; Su et al. 1998; Cultrera et al. 1999). In those studies, the nonlinear effect causes the reduction of waveform amplitude in the time domain and the shifting of predominant frequencies and peak reduction in the frequency domain. They are due to the nonlinear response of the material that causes change in the elastic properties of the medium dependent on the waveform amplitudes. Aguirre and Irikura (1997) studied the nonlinearity, liquefaction, and velocity variation of soft soil layers in Port Island, Kobe, during the 1995 Hyogo-ken Nanbu earthquake. The S-wave velocity structure before and after the mainshock was found to be different. Specifically, the S-wave velocity of the second layer (5 to $16 \mathrm{~m}$ depths) after the mainshock was $20 \%$ lower than before. The liquefied state remains at least $3 \mathrm{hr}$ after the mainshock but no more than $24 \mathrm{hr}$. The strong influence of nonlinearity during the mainshock yielded a significant reduction (25\%) of the horizontal ground motions at surface.

Several techniques have been used to detect the nonlinear effect. One is spectral ratio evaluation of the observed data between surface and bedrock during strong and weak ground motions (Ordas and Faccioli 1994; Beresnev et al. 1995a; Hartzell 1998; Su et al. 1998). Another option for estimating the soil layer effect is to use the recordings from a vertical array of seismometers (Wen et al. 1994, 1995; Aguirre and Irikura 1995; Beresnev et al. 1995b; Satoh et al. 1995; Borja et al. 1999, 2000). The reduction and / or shift of the peaks during strong motion are indications of the nonlinearity. Another technique used to evaluate nonlinearity is based on the comparison of the observed ground motions during strong motion with those simulated by a linear method. The difference with the observed data can be interpreted as nonlinearity. Two commonly used linear methods are the 1D Haskell method and the empirical Green's function method (e.g., Aki and Irikura 1991; Aguirre et al. 1994). Numerical approaches to predict the nonlinear response of soil can be classified as either an equivalent secant approach (e.g., the SHAKE program by Schnabel et al. 1972) or a direct nonlinear approach (e.g., the DESRA-2 program by Lee and Finn 1982, the CHARSOIL program by Streeter et al. 1974 and the SPECTRA program by Borja and Wu 1994). Yu et al. (1993) used a direct nonlinear approach, DESRA-2, to examine the differences between linear and nonlinear soil response with various levels of base excitations. They showed that in strong excitation the soil nonlinearity causes deamplification and also a shift in peak frequencies to lower values for an unsaturated shallow soil deposit of $20 \mathrm{~m}$ thickness. Ni et al. (1997) used a modified version of DESRA-2 constitutive model for saturated soil to study the nonlinear seismic response including liquefaction of medium dense soil deposits of various thicknesses. By using the stress-dependent model with impulse base excitation, the nonlinear behavior of various soil deposit shows lower deamplification and higher first-mode (resonant) frequency than that of the stress-independent soil properties model.

In this article, we use the recordings at the Lotung vertical array to study the linear and 
nonlinear behavior of soft soil layers. First, the spectral ratio is calculated by dividing the spectrum at surface relative to that at the depths of $6,11,17$ and $47 \mathrm{~m}$ for the strong motion and weak motion events. Based on the shear-wave velocity structure by uphole and crosshole shooting methods, we simulate the 20 May 1986 earthquake sequence including the foreshock, aftershock and mainshock events for the linear and nonlinear models. The nonlinear numerical scheme, DESRA-2, is also used to predict the ground accelerations of the strong motion events.

\section{SITE AND DATA}

The downhole array (represented by the star symbol in Fig. 1) is in the Lanyang alluvial deposits at the LSST (Lotung large-Scale Seismic Test) site in the southwest quadrant of the SMART1 array, Lotung in northeastern Taiwan. The sediments are mostly composed of interlayered silty sand and silty clay bed with gravel (Anderson and Tang 1989). This site is geotechnically classified as "deep cohesionless soil site". A geophysical measurement program was conducted to determine P-wave and S-wave velocities (HCK 1986; Anderson and Tang 1989). The shallow S-wave velocities are estimated by crosshole and uphole shooting methods and tabulated in Table II of Wen's paper (Wen 1994). Shear wave velocity increases gradually from $120 \mathrm{~m} / \mathrm{sec}$ at the ground surface to $190 \mathrm{~m} / \mathrm{sec}$ at the depths of $8-13 \mathrm{~m}$. Below this depth, the shear wave velocity increases gradually to approximately $220-280 \mathrm{~m} / \mathrm{sec}$ at the depth of $60 \mathrm{~m}$. The results of up-hole testing indicate that shear wave velocities are $320 \mathrm{~m} / \mathrm{sec}$

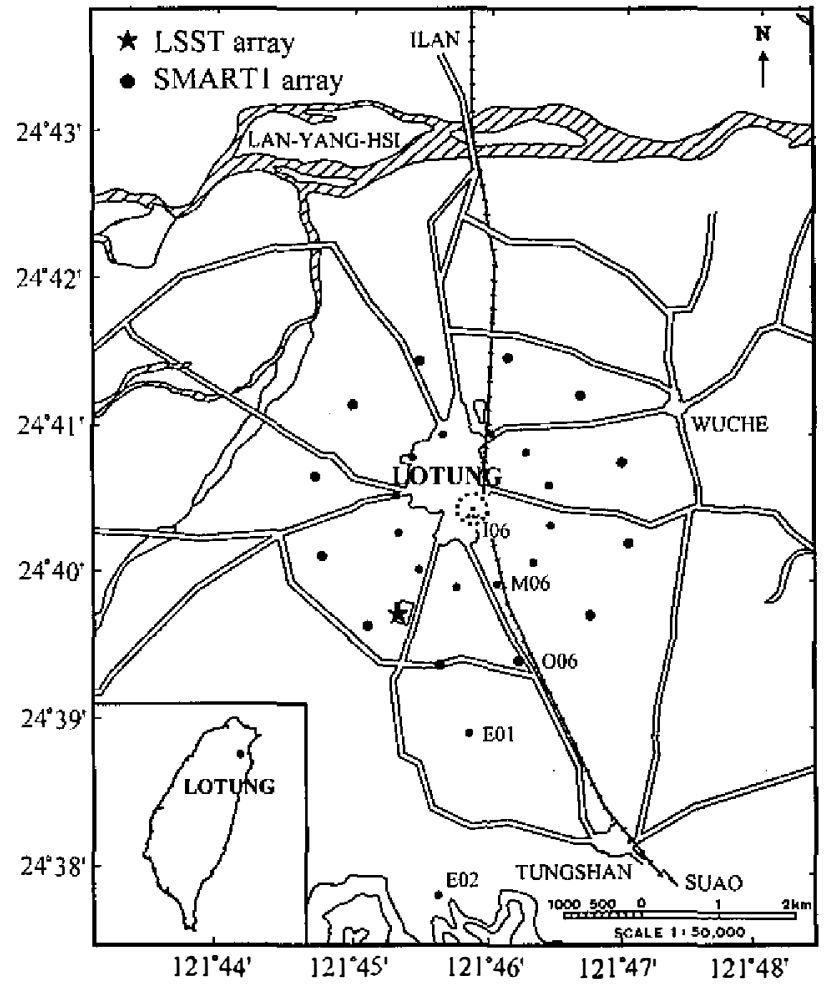

Fig. 1. A map shows the location of the Lotung downhole array (star symbol) that is inside the LSST (Lotung largeScale Seismic Test) site in the Lanyang alluvial plain. The site is in the southwest quadrant of the SMART1 array, Lotung, northeastern Taiwan. 
at the depths of $60-80 \mathrm{~m}$ and $480 \mathrm{~m} / \mathrm{sec}$ at the depths of $80-150 \mathrm{~m}$.

Two vertical arrays, DHA and DHB, were deployed around the building of the 1/4-scale nuclear plant containment structure. A schematic cross-section of the site, the deployment of instruments and the velocity profiles of the shear wave measured by uphole and crosshole shooting methods are shown in Fig. 2. Due to the proximity of the DHA array to the structure ( $3.2 \mathrm{~m}$ ), we use the data recorded by the DHB array. The DHB array is composed of six triaxial accelerometers deployed at the depths of $0,6,11,17,47$ and $93 \mathrm{~m}$ represented by FAl5, DHB6, DHB11, DHB17, DHB47 and DHB93 individually. The instrument at the $93 \mathrm{~m}$ depth did not catch any events, therefore, only the recordings at five depths are discussed here. Digital data were recorded as 12-bit words at the rate of 200 samples per second. During a 6year operation from 1985 to 1990,30 earthquakes $\left(4.0 \leq M_{L} \leq 6.5\right)$ riggered this array. Figure 3 shows the epicentral locations of these earthquakes. The relative ground motion parameters, PVA and PHA values at the surface station FA1-5 for these 30 earthquakes are tabulated in Table 1. The maximum PGA (peak ground acceleration) value is 258 gals recorded at the surface station FA 1-5 (Event no. 4). Here events with peak ground acceleration at the surface larger than 150 gals are attributed to the "strong motion" and less than 100 gals are considered as the "weak motion". The ground acceleration between the two classes is the ransition zone

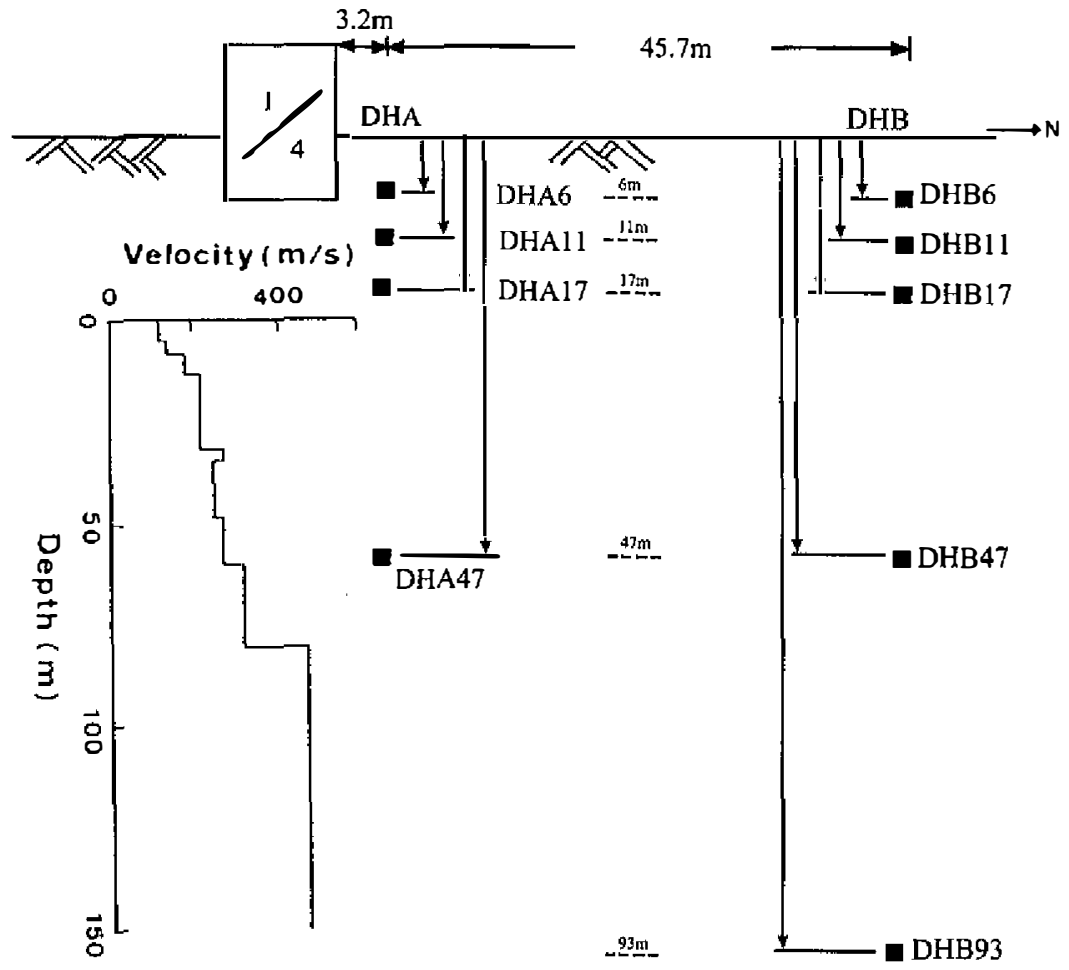

Fig. 2. A schematic cross-section of the site, the deployment of instruments at the depths of $0,6,11,17$ and $47 \mathrm{~m}$ and the velocity profiles of the shear wave. 
where the nonlinear effect begins.

On spectral ratio analysis, a 5 or 10-sec window, which primarily brackets the large amplitude S-wave parts of seismograms, is used with a 5\% cosine taper. We choose the 10-sec window for strong motion and the 5-sec window for weak motion. Fourier amplitudes are calculated for all accelerograms used. In order to avoid the pseudo-peak, twenty consecutive smoothing using a 3-point running Hanning average is applied to the raw spectra and then spectral ratios are calculated. We chose 19 events including 4 strong motion events (marked with " $S$ " in Table 1) and 15 weak motion events (marked with "W" in Table 1) based on record quality and completeness. Since all the waveforms used are near-field recordings, the signalnoise ratios are all above a factor of 10 that errors in the spectra introduced by the noise may be insignificant.

\section{TECHNIQUES}

Site effect can be considered as empirical transfer functions of the surficial layers. Several methods have been used to study the problem. Two techniques commonly used are the standard spectral ratio and the numerical simulations. Both are considered here and their results compared in the evaluation of the site effect. The standard spectral ratio, $S_{T}$, can be calculated by dividing the horizontal Fourier spectrum of the ground motions recorded on an alluvium site, $S_{H S}$, by that recorded on a nearby rock site or at the downhole, $S_{H B}$.

$$
S_{T}=\frac{S_{H S}}{S_{H B}} \text {. }
$$

The latter station is considered to be a reference station. The spectral ratios are taken at surface relative to the different depths of $6,11,17$ and $47 \mathrm{~m}$. Basically, the ratios have some variations due to the difference of the incidence angles and the heterogeneity of the medium. The transfer function could be considered as an average of ratios for several events at each depth.

For the numerical simulations, they are divided into two parts. One is the 1D Haskell method (Haskell 1953, 1960, 1962), based on the linear model, for the weak motions. Huang (1994) revised the technique to simulate the ground motions at the different depths using that at surface. The best advantage is that the upgoing and downgoing waves need not to be distinguished and separated from the input motion. It can be used to directly simulate the whole waveform at downhole. The other is the DESRA-2 numerical scheme, based on the nonlinear model, for the strong motions.

\subsection{Haskell Method (SH wave)}

For a given laterally homogeneous layered medium, if the thickness and the shear velocity of the medium of layer $\mathrm{m}$ are $d_{m}$ and $\beta_{m}$, the ground motions at the bottom and the top of layer $m$ can be correlated by a matrix (Haskell 1960)

$$
a_{m}=\left(\begin{array}{cc}
\cos Q_{m} & i \mu_{m}^{-1} \gamma_{\beta m}^{-1} \sin Q_{m} \\
i \mu_{m} \gamma_{\beta m} \sin Q_{m} & \cos Q_{m}
\end{array}\right),
$$




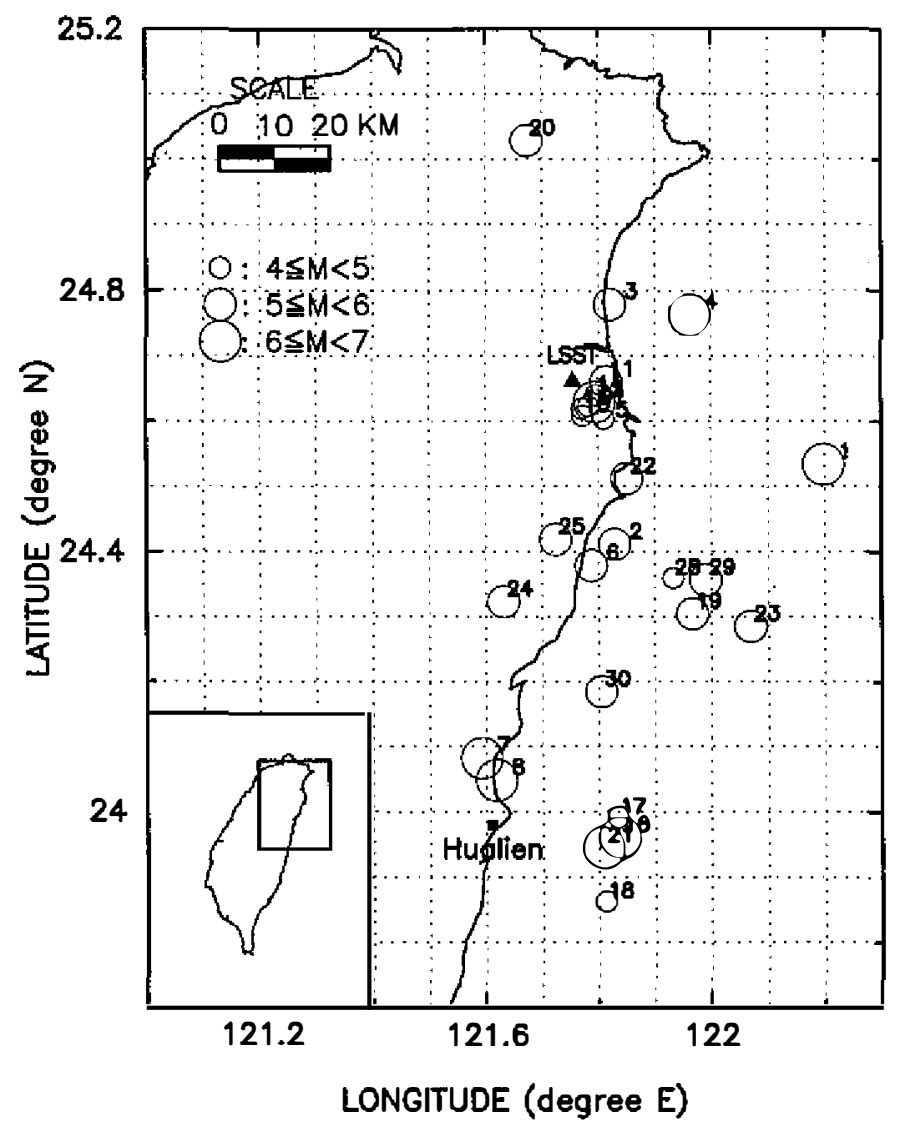

Fig. 3. Map showing the epicenters of 30 events $\left(4.0 \leq M_{L} \leq 6.5\right)$ triggering this downhole array during a 6-year operation from 1985 to 1990.

where $i=\sqrt{-1}, \mu$ is the shear modulus, $\gamma_{\beta}=\left[(c / \beta)^{2}-1\right]^{1 / 2}$ and $Q=k d \gamma_{\beta} . c$ in $\gamma_{\beta}$ is the horizontal phase velocity, and $k$ in $Q$ is the apparent wave number. If $v$ represents the transverse component of displacement and $Y_{z}$ stands for the transverse shear stress, the ground motion and the stress between the underground interface $n$ and the free surface become

$$
\left(\begin{array}{l}
\frac{\dot{v}}{c} \\
Y_{z}
\end{array}\right)_{n-1}=[A]\left(\begin{array}{l}
\frac{\dot{v}}{c} \\
Y_{z}
\end{array}\right)_{0},
$$

where $[A]$ is a $2 \times 2$ matrix given by taking the product of all the propagating matrix between the free surface and the $n^{\text {th }}$ interface, i.e.

$$
[A]=a_{n-1} a_{n-2} \ldots a_{2} a_{1} .
$$

On the free surface, the shear stress vanishes and (3) can be further simplified as 
Table 1. The source parameters and PGA values of 30 earthquakes recorded at LSST array site.

\begin{tabular}{|c|c|c|c|c|c|c|c|c|c|c|}
\hline $\begin{array}{c}\text { EQ } \\
\text { type }\end{array}$ & $\begin{array}{c}\text { Event } \\
\text { no. }\end{array}$ & $\begin{array}{l}\text { Earthquake original time } \\
\text { (yr/mon/day hr:min:sec) }\end{array}$ & $\begin{array}{l}\text { Latitude } \\
\text { ('N) }\end{array}$ & $\begin{array}{l}\text { Longitude } \\
\left({ }^{\circ} \mathrm{E}\right)\end{array}$ & $\begin{array}{l}\text { Focal } \\
\text { depth } \\
(\mathrm{Km})\end{array}$ & $\mathrm{M}_{\mathrm{L}}$ & $\begin{array}{c}\Delta \\
(\mathrm{Km})\end{array}$ & $\begin{array}{l}\theta \\
\left({ }^{\circ}\right)\end{array}$ & $\begin{array}{c}\text { PVA } \\
\text { of FAI-5 } \\
\left(\mathrm{cm} / \mathrm{sec}^{2}\right)\end{array}$ & $\begin{array}{c}\text { PHA } \\
\text { of FAl }-5 \\
\left(\mathrm{~cm} / \mathrm{sec}^{2}\right)\end{array}$ \\
\hline & 1 & $1985 / 09 / 20 \quad 15: 01: 24.00$ & 24-31.97 & $122-11.87$ & 6.1 & 6.3 & 45.7 & 107.4 & & \\
\hline & 2 & 1985/10/26 $03: 30: 39.10$ & $24-24.67$ & $121-49.70$ & 1.7 & 5.3 & 29.1 & 164.5 & & \\
\hline & 3 & $1985 / 11 / 07 \quad 05: 25: 17.84$ & $24-46.69$ & $121-49.19$ & 73.8 & 5.5 & 14.6 & 30.5 & 6.7 & 24.9 \\
\hline S & 4 & 1986/01/16 $13: 04: 31.97$ & $24-45.77$ & $121-57.67$ & 10.2 & 6.5 & 23.8 & 61.3 & 104.3 & 258.0 \\
\hline W & 5 & 1986/03/29 07:17:14.66 & $24-36.16$ & $121-48.59$ & 10.3 & 4.7 & 8.4 & 133.9 & 32.5 & 41.5 \\
\hline W & 6 & 1986/04/08 02:14:58.51 & $24-22.72$ & $121-47.19$ & 10.9 & 5.4 & 31.3 & 174.2 & 11.2 & 35.4 \\
\hline $\mathrm{s}$ & 7 & $1986 / 05 / 20 \quad 05: 25: 49.58$ & 24-04.90 & $121-35.49$ & 15.8 & 6.5 & 66.2 & 194.5 & 40.0 & 203.7 \\
\hline W & 8 & $1986 / 05 / 20 \quad 05: 37: 31.69$ & $24-0289$ & $121-37.04$ & 21.8 & 6.2 & 69.2 & 191.7 & 9.7 & 34.3 \\
\hline W & 9 & $1986 / 07 / 11 \quad 18: 25: 26.27$ & $24-37.35$ & $121-46.96$ & 1.1 & 4.5 & 5.0 & 146.1 & 14.1 & 70.6 \\
\hline W & 10 & $1986 / 07 / 16 \quad 23: 50: 31.84$ & $24-36.47$ & $121-46.35$ & 1.0 & 4.5 & 6.0 & 163.0 & 15.8 & 39.4 \\
\hline W & 11 & $1986 / 07 / 17 \quad 00: 03: 33.51$ & 24-39.59 & $121-48.89$ & 2.0 & 5.0 & 6.0 & 90.1 & 43.2 & 101.7 \\
\hline \multirow[t]{2}{*}{$\mathrm{S}$} & 12 & 1986/07/30 $11: 31: 47.53$ & $24-37.73$ & $121-47.65$ & 1.6 & 6.2 & 5.2 & 131.1 & 191.8 & 186.7 \\
\hline & 13 & $1986 / 07 / 30 \quad 11: 32: 00.53$ & 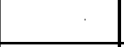 & & & & & & 17.4 & 48.7 \\
\hline W & 14 & $1986 / 07 / 30 \quad 11: 38: 31.70$ & $24-38.38$ & $121-47.73$ & 2.3 & 4.9 & 4.7 & 118.8 & 20.9 & 49.4 \\
\hline W & 15 & $1986 / 08 / 05 \quad 00: 56: 23.50$ & $24-37.13$ & $121-46.30$ & 1.2 & 4.9 & 4.9 & 159.8 & 26.4 & 98.1 \\
\hline$S$ & 16 & 1986/11/14 21:20:01.20 & $23-57.65$ & $121-50.17$ & 6.9 & 6.8 & 77.9 & 173.9 & 95.9 & 167.3 \\
\hline \multirow[t]{4}{*}{ W } & 17 & $1986 / 11 / 14 \quad 23: 04: 00.00$ & $23-59.51$ & 121.49 .99 & 15.0 & 4.0 & 77.9 & 173.9 & 16.5 & 37.0 \\
\hline & 18 & $1986 / 11 / 15 \quad 00: 18: 00.00$ & $23-51.76$ & $121-48.68$ & 7.6 & 4.0 & 77.9 & 173.9 & 6.7 & 28.0 \\
\hline & 19 & 1986/12/08 09:38:23.68 & 24-18.29 & $121-57.98$ & 26.7 & 5.8 & 44.8 & 151.4 & & \\
\hline & 20 & 1986/12/10 23:55:22.19 & $25-01.66$ & $121-40.39$ & 98.6 & 5.8 & 41.6 & 348.5 & 5.4 & 23.9 \\
\hline W & 21 & 1987/01/06 05:07:49.00 & $23-56.71$ & $121-48.46$ & 27.8 & 6.2 & 79.3 & 179.5 & 9.1 & 31.8 \\
\hline W & 22 & 1987/02/04 12:15:24.30 & |24-30.67 & $121-51.03$ & 70.1 & 5.8 & 19.1 & 181.6 & 7.7 & 43.2 \\
\hline W & 23 & 1987/06/24 22:38:38.90 & $24-17.12$ & $122-04.12$ & 30.6 & 5.7 & 52.3 & 142.5 & 5.0 & 31.6 \\
\hline W & 24 & 1987/06/27 07:38:55.70 & $24-19.41$ & $121-37.86$ & 0.5 & 5.3 & 39.3 & 199.8 & 11.9 & 23.8 \\
\hline \multirow[t]{2}{*}{ W } & 25 & $1987 / 11 / 10 \quad 04: 33: 09.21$ & $24-25.11$ & $121-43.42$ & 34.4 & 5.2 & 26.9 & 186.8 & 16.4 & 78.3 \\
\hline & 26 & |1988/08/19 18:10:08.65 & $|24-35.06|$ & $122-42.21$ & 91.2 & 5.9 & 96.4 & 95.0 & 13.5 & 22.3 \\
\hline \multirow[t]{4}{*}{ W } & 27 & 1988/09/18 $15: 38: 25.92$ & $24-28.71$ & $122-24.09$ & 62.5 & 5.6 & 68.5 & 107.1 & 6.8 & 22.3 \\
\hline & 28 & $1990 / 01 / 15 \quad 12: 42: 30.14$ & $24-21.58$ & $121-55.92$ & 20.1 & 4.8 & 37.8 & 151.7 & 22.5 & 65.4 \\
\hline & 29 & 1990/04/15 22:44:09.51 & $24-21.41$ & $121-59.40$ & 22.1 & 5.6 & 41.2 & 144.7 & 9.7 & 51.1 \\
\hline & 30 & 1990/07/16 19:14:52.00 & 24-11.04 & $121-48.24$ & 1.4 & 5.3 & 52.9 & 174.6 & 11.5 & 17.9 \\
\hline
\end{tabular}

NOTE : (1) EQ type : $\mathrm{S}$ and $\mathrm{W}$ represent strong and weak motion event.

(2) $\Delta$ : Distance between the Lotung LSST array and epicenters.

(3) $\theta:$ Back-azimuth.

(4) PVA \& PHA : Peak Vertical and Horizontal Acceleration. 


$$
\begin{aligned}
& \left(\frac{\dot{v}}{c}\right)_{n-1}=A_{11}\left(\frac{\dot{v}}{c}\right)_{0}, \\
& \left(Y_{z}\right)_{n-1}=A_{2 \mathrm{r}}\left(\frac{\dot{v}}{c}\right)_{0},
\end{aligned}
$$

where $A_{11}$ is the element of matrix $[A]$ at row 1 and column 1 . For a given subsurface structure, element $A_{11}$ is a very stable value even for a zero damping material. Therefore, (5) is a stable formula for calculating the downhole displacement using the surface displacement.

\subsection{DESRA-2 Numerical Scheme}

The program DESRA-2 developed by Lee and Finn (1982), that is a direct nonlinear computational method, used to calculate the nonlinear soil response. In the DESRA-2, the initial loading phase is defined by the hyperbolic stress-strain relationship

$$
\tau=\frac{G_{m o} \gamma}{1+\frac{G_{m o}}{\tau_{m o}}|\gamma|},
$$

in which $\gamma$ is the strain amplitude, $\tau$ is the corresponding shear stress, $G_{m o}$ and $\tau_{m o}$ are the initial maximum shear modulus and shear stress. Thus as the strain increases, the stress tending to restore the medium also increases but is asymptotic to a maximum level. For very large value of $\tau_{m o}$ the stress-strain relationship is approximately linear, while for smaller values of $\tau_{m o}$ the stress-strain relationship becomes more severely nonlinear as the strain amplitude $\gamma$ becomes larger. In the subsequent unloading and reloading phases, the stress-strain relationship is given by the Massing stress-strain curve (Masing 1926; Lee and Finn 1982) that is described by

$$
\frac{\tau-\tau_{r}}{2}=\frac{\frac{G_{m t}\left(\gamma-\gamma_{r}\right)}{2}}{1+\frac{G_{m s}}{2 \tau_{m t}}\left|\gamma-\gamma_{r}\right|},
$$

where $G_{m t}$ and $\tau_{m s}$ are the maximum shear modulus and shear stress during the unloading and reloading, which are changing continually due to the development of pore-water pressure and strain hardening during un loading and reloading. In this study, we ignored the effects of porewater pressure and strain hardening so that the maximum shear modulus $G_{m t}$ and shear stress $\tau_{m t}$ during the unloading and reloading are equivalent to the initial maximum shear modulus $G_{m o}$ and shear stress $\tau_{m o}$. Two parameters, $\tau_{r}$ and $\gamma_{r}$, are the shear stress and strain corresponding to the turning point of the hysteresis loop. As implemented in the program, a layered soil model, in which properties depend only on the depth, is excited by the horizontal shear wave propagating vertically.

According to Seed and Idriss (1970), the quantities $G_{m o}$ and $\tau_{m o}$ were given values that 
are representative of a medium dense sand with relative density of about $65 \%$. In units of $\mathrm{kN} / \mathrm{m}^{2}$, they can be expressed as

$$
G_{m o}=12016 \sqrt{\sigma_{m}^{\prime}}
$$

and

$$
\tau_{m o}=\left[\left(\frac{1+K_{0}}{2} \sin \phi^{\prime}\right)^{2}-\left(\frac{1-K_{0}}{2}\right)^{2}\right]^{1 / 2} \cdot \sigma_{v}^{\prime},
$$

where $\sigma_{m}^{\prime}$ is the mean principal effective stress, $\sigma_{v}^{\prime}$ is the vertical effective stress, $K_{0}$ is the coefficient of lateral stress at rest, and $\phi^{\prime}$ is the effective angle of shearing resistance. Values for $\phi^{\prime}$ and $K_{0}$ were selected to be $35^{\circ}$ and 0.43 , which are typical values for medium dense sand. The mean effective stress can be computed as

$$
\sigma_{m}^{\prime}=\frac{1+2 K_{0}}{3} \sigma_{v}^{\prime} .
$$

The parameter, $\tau_{m o}$, is related to the strength envelope of the soil and can be easily derived from a Mohr circle failure envelope as given by equation (9).

\section{SPECTRAL ANALYSIS}

The results of spectral ratio analysis are described first. We calculate spectral ratios at surface relative to the depths of $6,11,17$ and $47 \mathrm{~m}$. Figure 4 shows the RMS results during the May 20, 1986, earthquake sequence (No. 6, 7 and 8 tabulated in Table 1). RMS is the root mean square of two horizontal component results. The thin, thick and dot-thin lines individually represent the spectral ratio of the foreshock (No. 6), mainshock (No. 7) and aftershock events (No. 8) of this strong motion sequence. Compared with foreshock $(4.5 \mathrm{~Hz}, 3.0 \mathrm{~Hz}, 2.2 \mathrm{~Hz}$ and $1.3 \mathrm{~Hz})$ and aftershock $(4.2 \mathrm{~Hz}, 2.9 \mathrm{~Hz}, 2.2 \mathrm{~Hz}$ and $1.2 \mathrm{~Hz})$, mainshock event $(2.8 \mathrm{~Hz}, 1.7 \mathrm{~Hz}$, $1.2 \mathrm{~Hz}$ and $0.85 \mathrm{~Hz}$ ) apparently has lower fundamental predominant frequency and smaller amplification factor. Four frequencies in the parentheses represent the fundamental predominant frequency at surface relative to the depths of $6,11,17$ and $47 \mathrm{~m}$ for that event. Many researchers attribute the phenomenon to the nonlinear behavior of soil after a large earthquake. It is similar to the theoretical calculation predicted by Yu et al. (1993). Besides, for the aftershock event, in 12 minutes after mainshock, the soil property is recovering but its predominant frequency at the first layer is still lower than that at the foreshock event. It probably can be explained that the retum time, only 12 minutes, was too short to go back to the initial state of the soil and then the aftershock followed closely. Furthermore, to investigate the changes of site responses between the strong motion and weak motion, we chose 15 weak motion events and the average spectral ratios of them are as the reference. Figure 5 shows the comparison between the spectral ratio of the mainshock (thin line) and the average spectral ratio of the 15 weak motion events (thick line) at surface relative to the depths of $6,11,17$ and $47 \mathrm{~m}$. The light thin lines represent one positive and negative standard deviation on the average weak- 


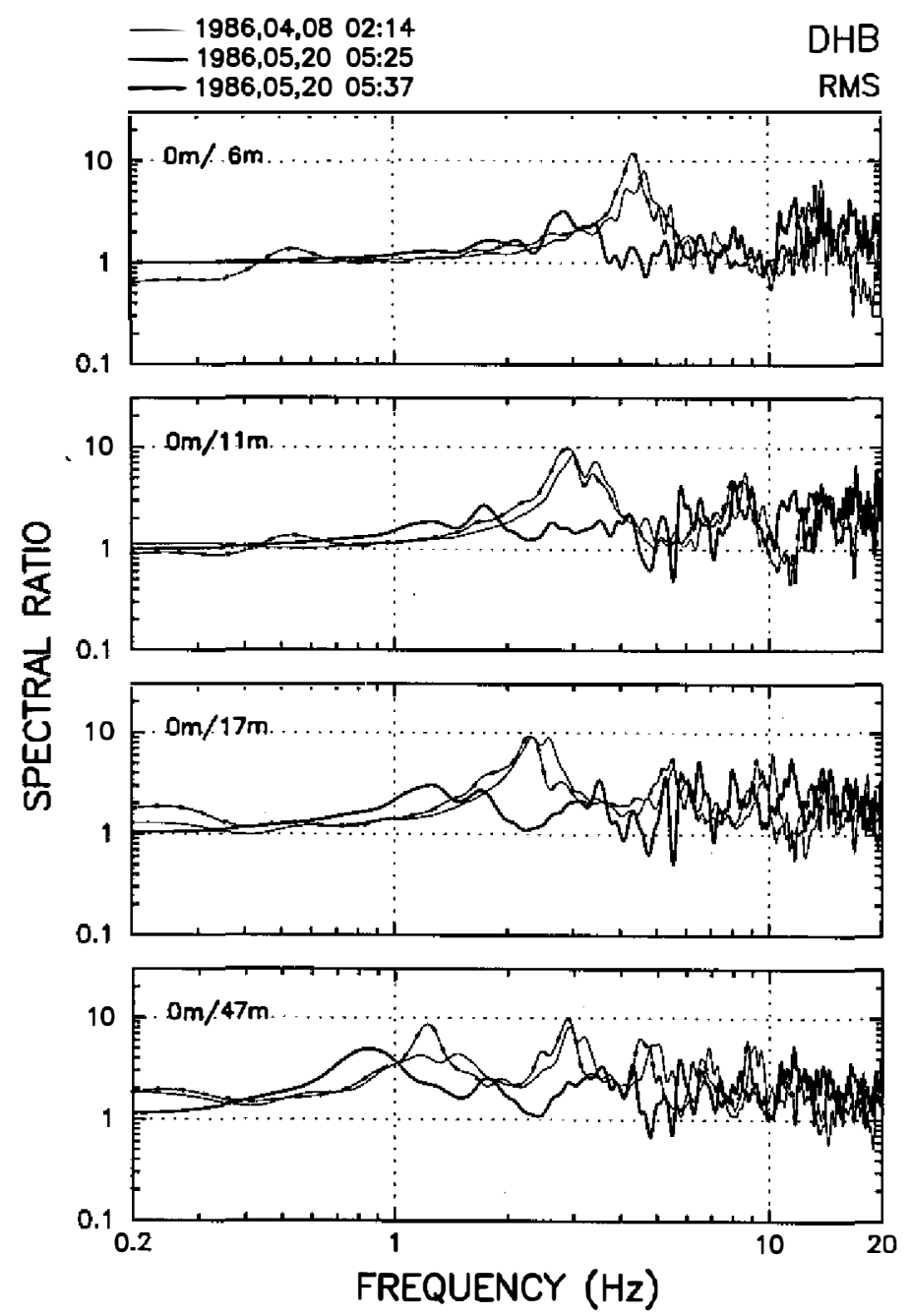

Fig. 4. The RMS spectal ratios at surface relative to the depths of $6,11,17$ and $47 \mathrm{~m}$ for the May 20, 1986 earthquake sequence (No. 6, 7 and 8). The thin, thick and dot-thin lines individually represent the foreshock (No. 6), mainshock (No. 7) and aftershock events (No. 8) for this strong motion sequence.

motion spectral ratio. For the weak motion events, the fundamental frequency at $0 / 6,0 / 11$, $0 / 17$ and $0 \mathrm{~m} / 47 \mathrm{~m}$ individually appears at $4.9 \mathrm{~Hz}, 3 \mathrm{~Hz}, 2.2 \mathrm{~Hz}$ and $1.2 \mathrm{~Hz}$. When the frequency band is less than $10 \mathrm{~Hz}$, the strong motion event has lower fundamental frequency than the weak motion events at the various depths. The largest shifting between them is about $20 \%$. The amplification factor also decreases for the strong motion event and the largest difference between strong and weak events is about $50 \%$. When the frequency range is between $10 \mathrm{~Hz}$ and $20 \mathrm{~Hz}$, the amplification factor of the strong motion becomes higher than the weak motions. 


\section{DHB}

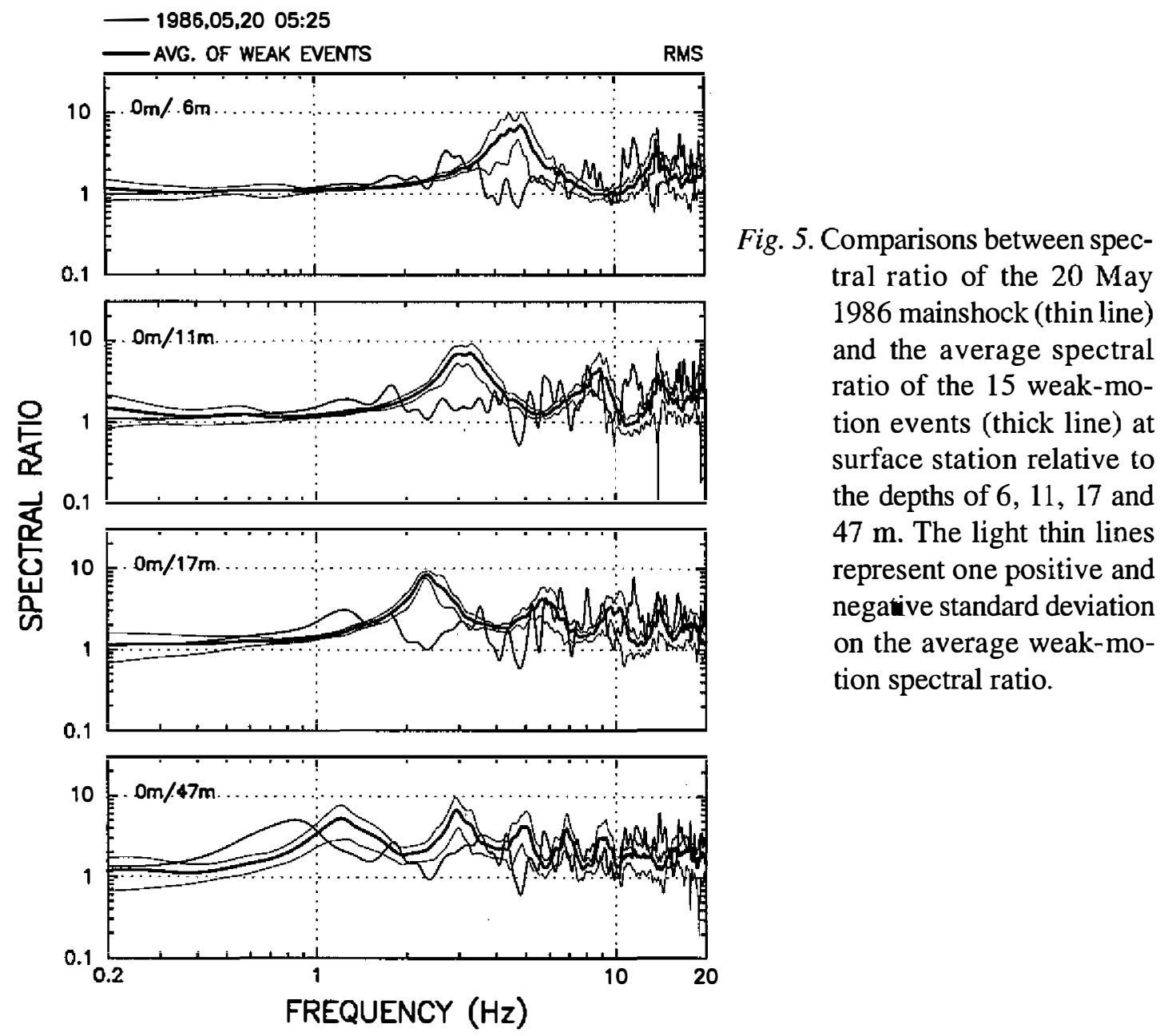

Besides, these two lines intersect at about $10 \mathrm{~Hz}$. The results are similar to Wen et al. (1994, 1995). Yu et al. (1993) also got the similar phenomenon using the theoretical simulation. It indicates that the strong motion event induces the nonlinear behavior of the soft soil at LSST array. Besides, the similar results also appear on the other three strong motion events (No. 4, 12 and 17) shown in Fig. 6 where the black thick line is also the average weak-motion spectral ratio and the other four lines represent the results of different strong motion events. It is apparent that all of these strong motion events have lower fundamental frequency than the weak motion events at the various depths.

\section{NUMERICAL SIMULATIONS}

In numerical simulation, it can be divided into two parts. One is the 1D Haskell method based on the linear model. Another one is for the nonlinear model, that is, the DESRA-2 


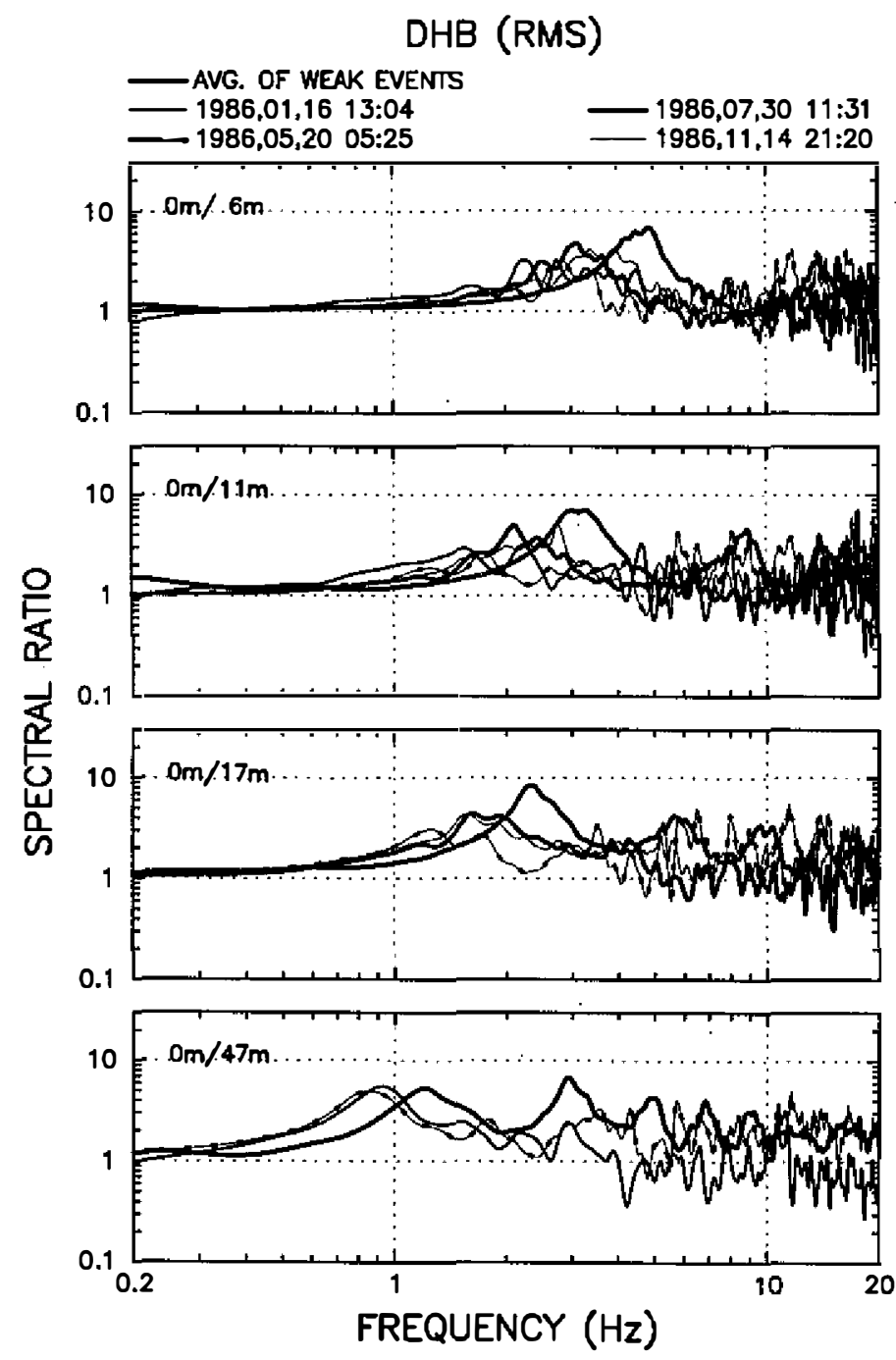

Fig. 6. Comparisons between four spectral ratios using strongmotion events (No. 4, 7, 12 and 17 ; represented by different kinds of lines) and the average spectral ratio of the 15 weak-motion events (black thick line) at surface station relative to the depths of $6,11,17$ and $47 \mathrm{~m}$.

numerical scheme. We will discuss them as the following.

\subsection{Linear Model}

The Lotung downhole array is located at the Lanyang alluvial plain. The shallow soil structure around the site can be represented by a horizontal layered structure (Wen and Yeh 1984). In order to study site amplification, we use the Haskell method (Haskell 1953, 1960, 1962; Huang and Chiu 1996) to simulate the ground motion of the horizontal and layered structure at the Lotung downhole site. The ground motion at the surface site is chosen as the input to predict those at the depths of $6,11,17$ and $47 \mathrm{~m}$. The great advantage of this approach is that the upgoing (or direct $S$ ) wave need not be separated from the downgoing (or reflected) wave. By means of this technique, the whole waveform corresponding to each layer can be 
calculated directly. According to Wen (1994), the used velocity structure and density are listed in Table 2 . No attenuation term $\left(1 / Q_{s}=0\right)$ is added to the simulation process. This is mainly because the propagating distance is short $(47 \mathrm{~m})$ and the bandpass used has removes high frequency components which would be most affected by $Q_{s}$.

In this part, the recordings of the 20 May 1986 earthquake sequences are analyzed. According to the back-azimuths of the earthquakes, two horizontal components are used to construct the SH wave. The velocity structure is divided into 11 layers and is considered to be at the half-space under the $47 \mathrm{~m}$ depth. It is assumed that the SH wave is vertically incident from the half-space. The ground motion at surface site is used to predict those at downholes. Figure 7 shows the synthetic results (thick lines) compared with the observed data (thin lines) for (a) the 29 March 1986 earthquake and (b) the 8 April 1986 earthquake. The synthetic waveforms at the depths of $6 \mathrm{~m}, 11 \mathrm{~m}, 17 \mathrm{~m}$ and $47 \mathrm{~m}$ match the observed in amplitude and in phase. The simplified model provides a good simulation for the weak ground motion recordings. However, for the aftershock event (No. 8), the synthetic waveforms match the observed one neither in amplitude nor in phase (Fig. 8a). This indicates that the shallow soil behavior may be changing. The fundamental resonance frequency of one layer is $f=v_{s} / 4 H$, where $v_{s}$ is the $\mathrm{S}$ wave velocity and $H$ is the layer thickness. According to this formula, we can roughly estimate the predominant frequency for the horizontal layered model. Therefore, a modified S-wave velocity structure is constructed. That is the $S$ wave velocity at the first layer is adjusted from $120 \mathrm{~m} / \mathrm{sec}$ to $95 \mathrm{~m} / \mathrm{sec}$. Figure $8 \mathrm{~b}$ shows the synthetic waveforms for the modified velocity structures. Both fit better in amplitude and in phase. But for the mainshock event (No. 7), the original model (Table 2) does not produce a simulation of the strong motion event that matches the data well (Fig. 9). Especially at the depth of $47 \mathrm{~m}$, the synthetic result is bad. However, the result can not be improved by means of adjusting velocity or incident angles. It means that the linear model based on Haskell method does not work well for the strong ground accelerations (PGA > 150 gals). This phenomenon is probably caused by the nonlinear effect of the soft soil after strong

Table 2. Velocity structure used in Haskell method at the Lotung downhole array.

\begin{tabular}{|c|c|c|c|c|}
\hline $\begin{array}{c}\text { Layer } \\
\text { No. }\end{array}$ & $\begin{array}{c}\text { Thickness } \\
(\mathrm{m})\end{array}$ & $\begin{array}{c}V_{P} \\
(\mathrm{~m} / \mathrm{sec})\end{array}$ & $\begin{array}{c}V_{s} \\
(\mathrm{~m} / \mathrm{sec})\end{array}$ & $\begin{array}{c}\text { Density } \\
\left(\mathrm{g} / \mathrm{cm}^{3}\right)\end{array}$ \\
\hline 1 & 3 & 370 & 120 & 1.87 \\
\hline 2 & 2 & 370 & 120 & 1.87 \\
\hline 3 & 1 & 810 & 140 & 1.87 \\
\hline 4 & 2 & 810 & 140 & 1.87 \\
\hline 5 & 3 & 1270 & 190 & 1.87 \\
\hline 6 & 2 & 1270 & 190 & 1.87 \\
\hline 7 & 4 & 1330 & 220 & 1.87 \\
\hline 8 & 7 & 1330 & 220 & 1.87 \\
\hline 9 & 7 & 1330 & 220 & 1.87 \\
\hline 10 & 3 & 1330 & 280 & 1.90 \\
\hline 11 & 13 & 1250 & 250 & 1.90 \\
\hline
\end{tabular}




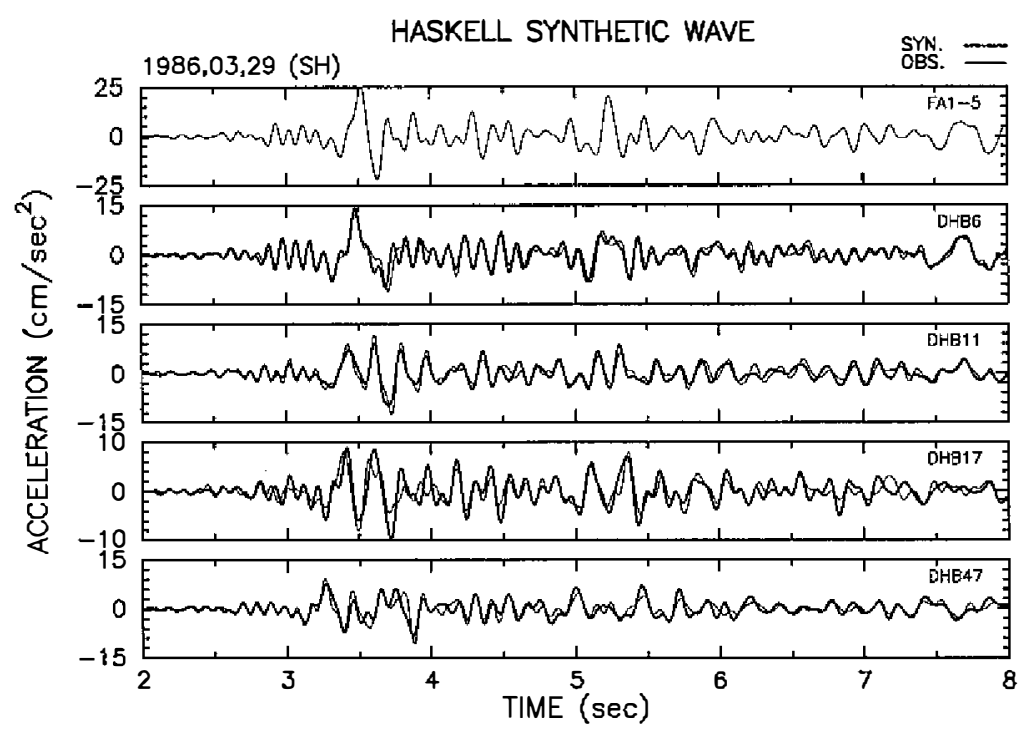

Fig. 7a. The synthetic ground acceleration (thick lines), using Haskell method, compared with the observed data (thin lines) at the Lotung downhole site in the 29 March 1986 earthquake (No. 5). The recording at surface is selected as the input motion and the $\mathrm{SH}$ wave is vertically incident from the half-space.

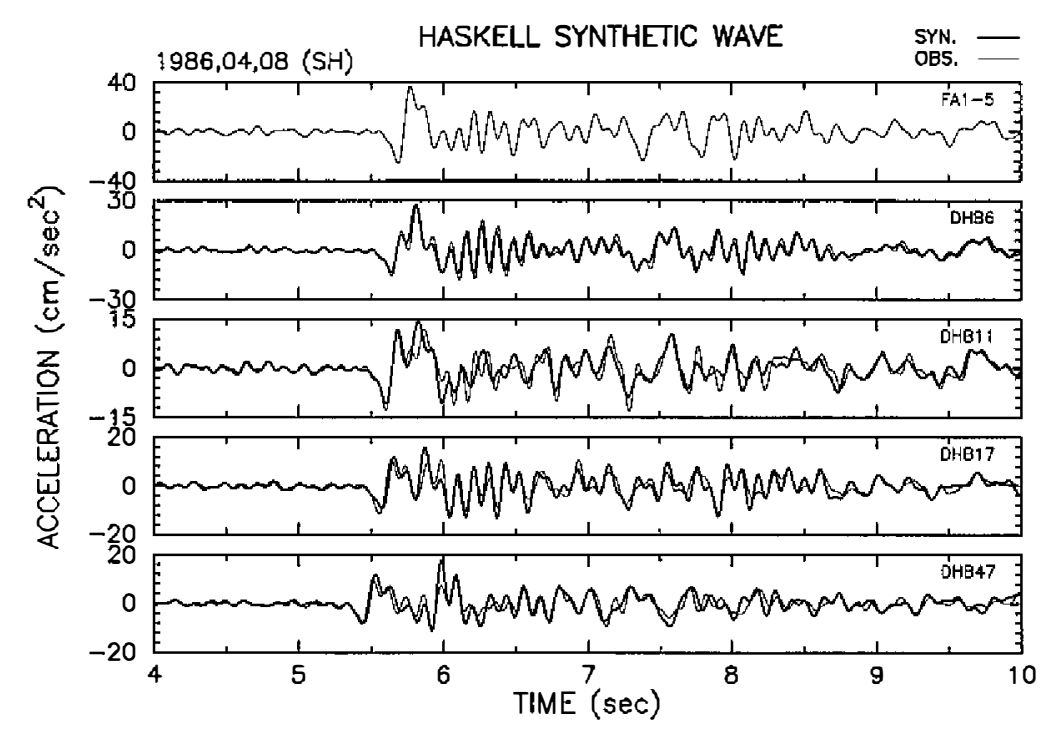

Fig. 7b. The synthetic ground acceleration (thick lines), using Haskell method, compared with the observed data (thin lines) at the Lotung downhole site in the 8 April 1986 earthquake (No. 6). The recording at surface is selected as the input motion and the $\mathrm{SH}$ wave is vertically incident from the half-space. 


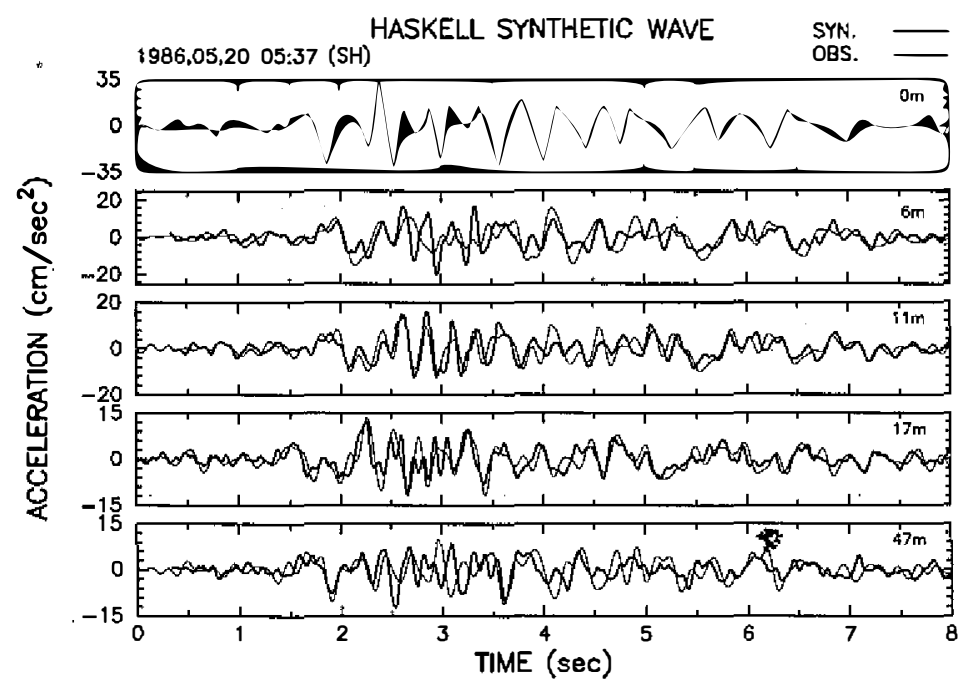

Fig. 8a. The synthetic ground acceleration (thick lines), using Haskell method with the velocity model in Table 2 , compared with the observed data (thin lines) at the Lotung downhole site in the 20 May 1986 aftershock event (No. 8). The recording at surface is selected as the input motion and the SH wave is vertically incident from the half-space.

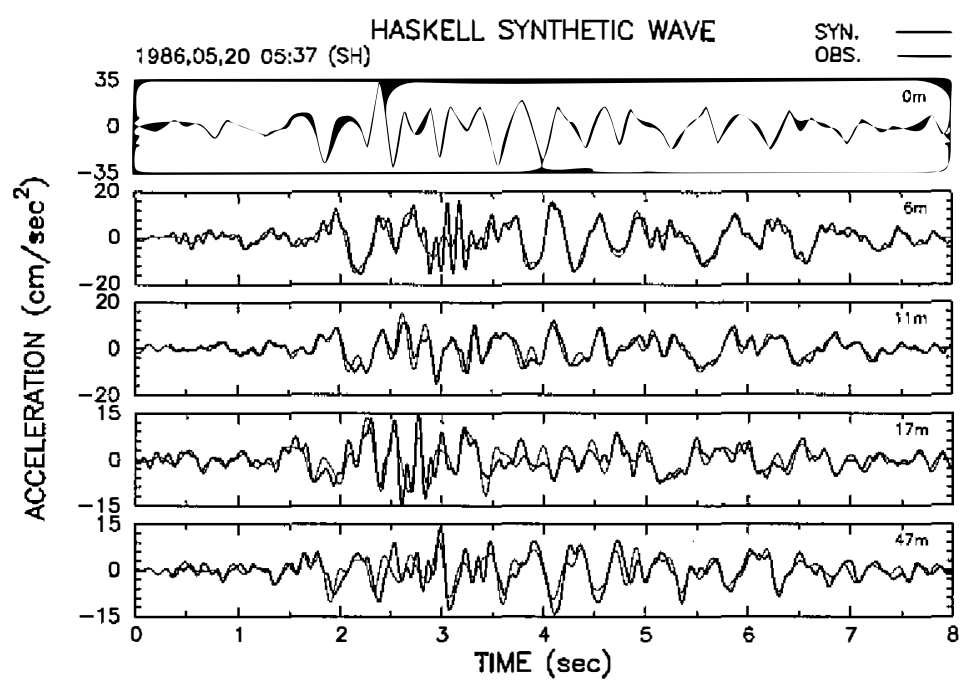

Fig. 8b. The synthetic ground acceleration (thick lines), using Haskell method with the modified velocity model where the velocity of the first layer is changed from 120 to $95 \mathrm{~m} / \mathrm{sec}$, compared with the observed data (thin lines) at the Lotung downhole site in the 20 May 1986 aftershock event (No. 8). The recording at surface is selected as the input motion and the $\mathrm{SH}$ wave is vertically incident from the half-space. 


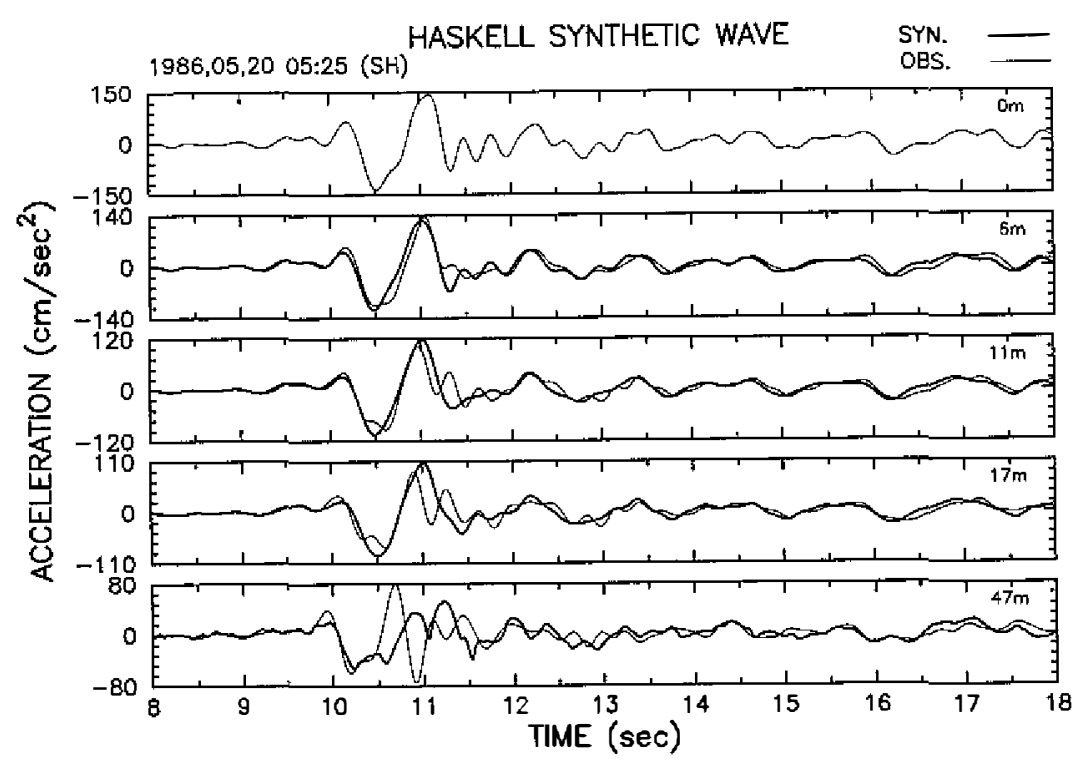

Fig. 9. The synthetic ground acceleration (thick lines), using Haskell method, compared with the observed data (thin lines) at the Lotung downhole site in the 20 May 1986 mainshock event (No. 7). The recording at surface is selected as the input motion and the $\mathrm{SH}$ wave is vertically incident from the half-space.

ground shaking. In order to solve this problem, the nonlinear numerical method (DESRA-2) is used next to predict the strong ground motions.

\subsection{Nonlinear Model}

To examine the DESRA-2 numerical technique, we did some tests to reproduce the results of Yu et al. (1993). According to Wen (1994), the velocity structures and some input parameters for the nonlinear model are listed in Table 3 where $G_{\max }=\rho v_{s}^{2}$, $\tau_{\max }=0.0005 G_{\max }$, and b value is relative to 5\% damping ratio ( $\mathrm{Yu}$ et al. 1993; $\mathrm{Ni}$ et al. 1997). First, the recording at the $47 \mathrm{~m}$ depth is selected to be the input motion to predict the ground motion at surface and other depths. Figure 10a shows the synthetic results (thick lines) compared with the observed data (thin lines) for the 20 May 1986 mainshock event (Table 1, No. 7). The amplitude at surface is underestimated about a factor of 0.5 , but they still have good fits in phase. It indicates that some errors appear in the velocity structure or the nonlinear behavior probably happens in the more shallow structure after strong motion. Therefore, we change the recording at the $17 \mathrm{~m}$ depth as the input motion. Figure $10 \mathrm{~b}$ shows the simulated results compared with the observed data. Apparently, there are better agreements in both amplitude and phase. We also get the stress-strain relationship and stress variation with time in this case. Figure 11 shows the stress-strain relationship and stress variation with time at the 10 $\mathrm{m}$ depth for the mainshock event. Before 10 seconds, the strain value is small and the stressstrain relationship is approximately linear (" $\mathrm{A}$ " part in Fig. 11a). As the strain becomes higher, 
Table 3. Velocity structure used in DESRA-2 scheme at the Lotung downhole array.

\begin{tabular}{|c|c|c|c|c|c|l|}
\hline $\begin{array}{c}\text { Layer } \\
\text { no. }\end{array}$ & $\begin{array}{c}\text { Thickness } \\
(\mathrm{m})\end{array}$ & $\begin{array}{c}V_{s} \\
(\mathrm{~m} / \mathrm{sec})\end{array}$ & $\begin{array}{c}\text { Density } \\
\left(\mathrm{g} / \mathrm{cm}^{3}\right)\end{array}$ & $\begin{array}{c}G_{\max } \\
(\mathrm{MPa})\end{array}$ & $\begin{array}{c}\tau_{\max } \\
(\mathrm{KPa})\end{array}$ & b values \\
\hline 1 & 5 & 120 & 1.87 & 26.93 & 13.46 & 0.0027 \\
\hline 2 & 3 & 140 & 1.87 & 36.65 & 18.33 & 0.0014 \\
\hline 3 & 5 & 190 & 1.87 & 67.51 & 33.75 & 0.0017 \\
\hline 4 & 18 & 220 & 1.87 & 90.51 & 45.25 & 0.0052 \\
\hline 5 & 3 & 280 & 1.90 & 148.96 & 74.48 & 0.0007 \\
\hline 6 & 13 & 250 & 1.90 & 118.75 & 59.38 & 0.0033 \\
\hline
\end{tabular}

NOTE : (1) b value represents about $5 \%$ damping ratio.

(2) $1 \mathrm{~Pa}=1 \mathrm{~N} / \mathrm{m}^{2}$

the stress-strain relationship follows the Masing curve (" $\mathrm{B}_{1}$ " and " $\mathrm{B}_{2}$ " part). The " $\mathrm{C}$ " point is where the maximum stress and the maximum strain happen at about 10 seconds. After that, the strain decreases again, the stress-strain relationship also turns to be a linear trend (" $\mathrm{D}$ " part). The level of strain value is between $-0.09 \% \sim 0.06 \%$. According to the above discussions, the

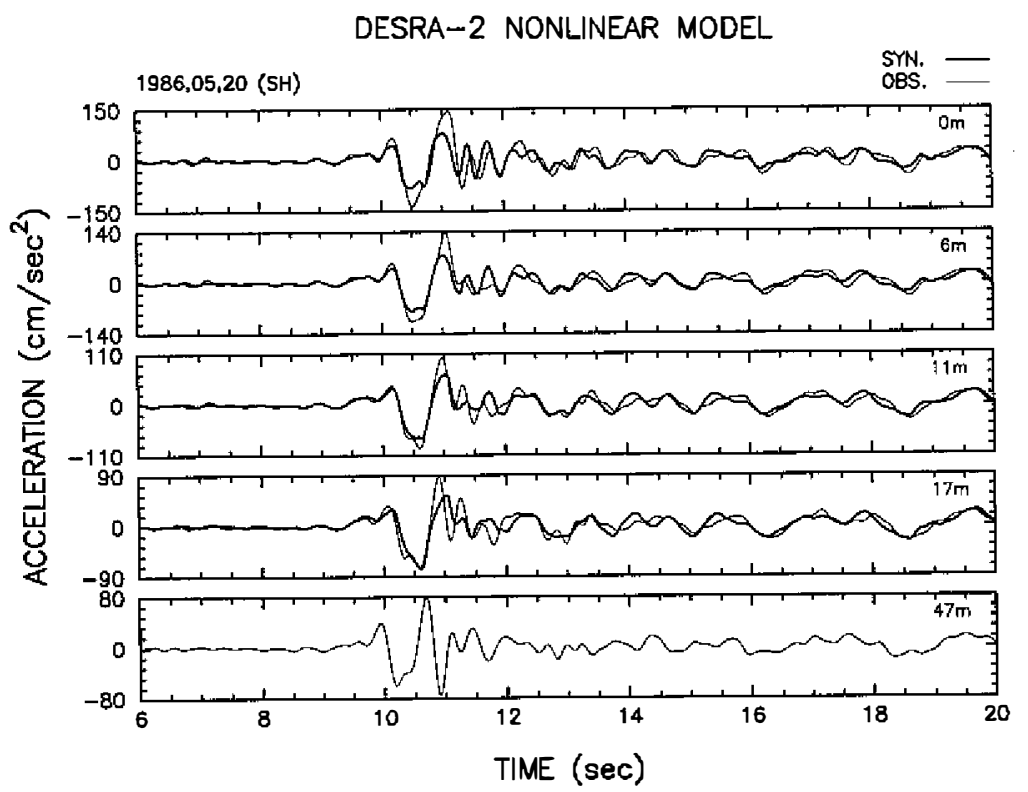

Fig. 10a. The synthetic ground acceleration (thick lines), using DESRA-2 numerical schedule, compared with the observed data (thin lines) at the Lotung downhole site in the 20 May 1986 mainshock event (No. 7). The recording at the $47 \mathrm{~m}$ depth is selected as the input motion. 


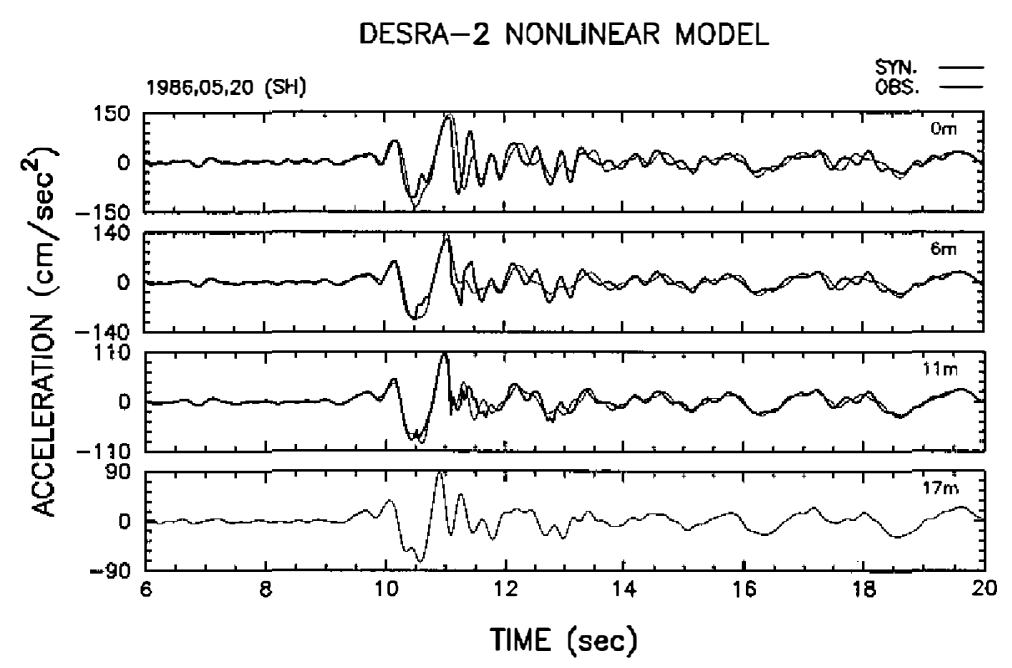

Fig. 10b. The synthetic ground acceleration (thick lines), using DESRA-2 numerical schedule, compared with the observed data (thin lines) at the Lotung downhole site in the 20 May 1986 mainshock event (No. 7). The recording at the $17 \mathrm{~m}$ depth is selected as the input motion.

soil has produced evident strain changes during the mainshock event. Therefore, the nonlinear numerical scheme, DESRA-2, is required and can significantly improve the simulation results although the PGA value at the surface station is still underestimated. Overall, it is feasible to predict the strong motions for a horizontal layered structure.

\section{CONCLUSIONS}

In order to study the linear and nonlinear behavior of the soft soil layers, the ground accelerations recorded by the DHB vertical array at LSST site in Lotung are analyzed in the paper. There are 30 earthquakes detected by this array during a 6-year operation. The maximum PGA value is 258 gals recorded at the surface station. Spectral ratios at surface relative to the depths of $6,11,17$ and $47 \mathrm{~m}$ are calculated. Spectral analyses show that the strong motion (PGA > 150 gals) causes the peaks of ratios to shift to lower predominant frequencies. Many researchers attribute the phenomenon to the nonlinear behavior of soil after a large earthquake. When the averaged spectral ratios of 15 well-recorded weak motion data (PGA < 100 gals) are selected as a reference, the shift of the maximum predominant frequency can reach $20 \%$. In comparison with the weak motions, the strong motions also decrease the amplification factor. The maximum reduction of the amplification can reach 50\%. It indicated that the strong motion events have induced the nonlinear effect of the soft layer at LSST array.

The shallow structure around the LSST site can be represented by a horizontal layered structure. The SH wave is vertically incident. The results of waveform simulation show that the linear model based on the Haskell method can well predict the weak motions at various 
(a)

STRESS - STRAIN RELATIONSHIP

$86,05,20$ 05:25 DEPTH OF $10 \mathrm{~m}$

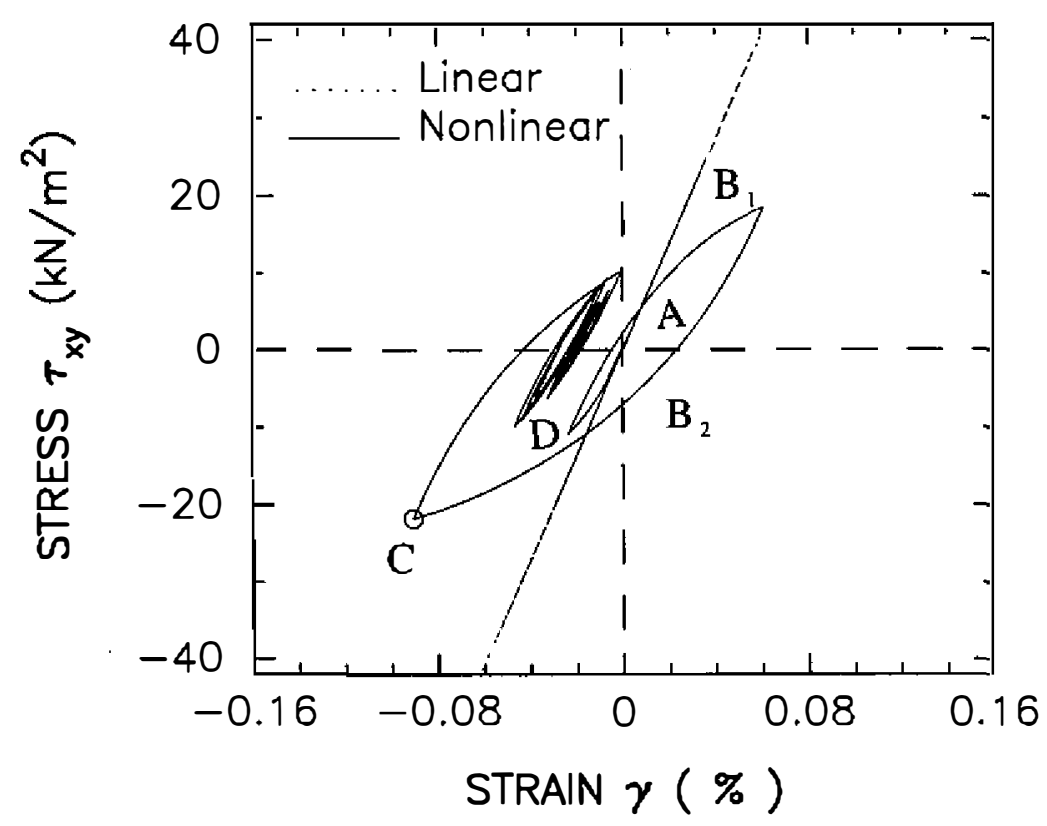

(b)

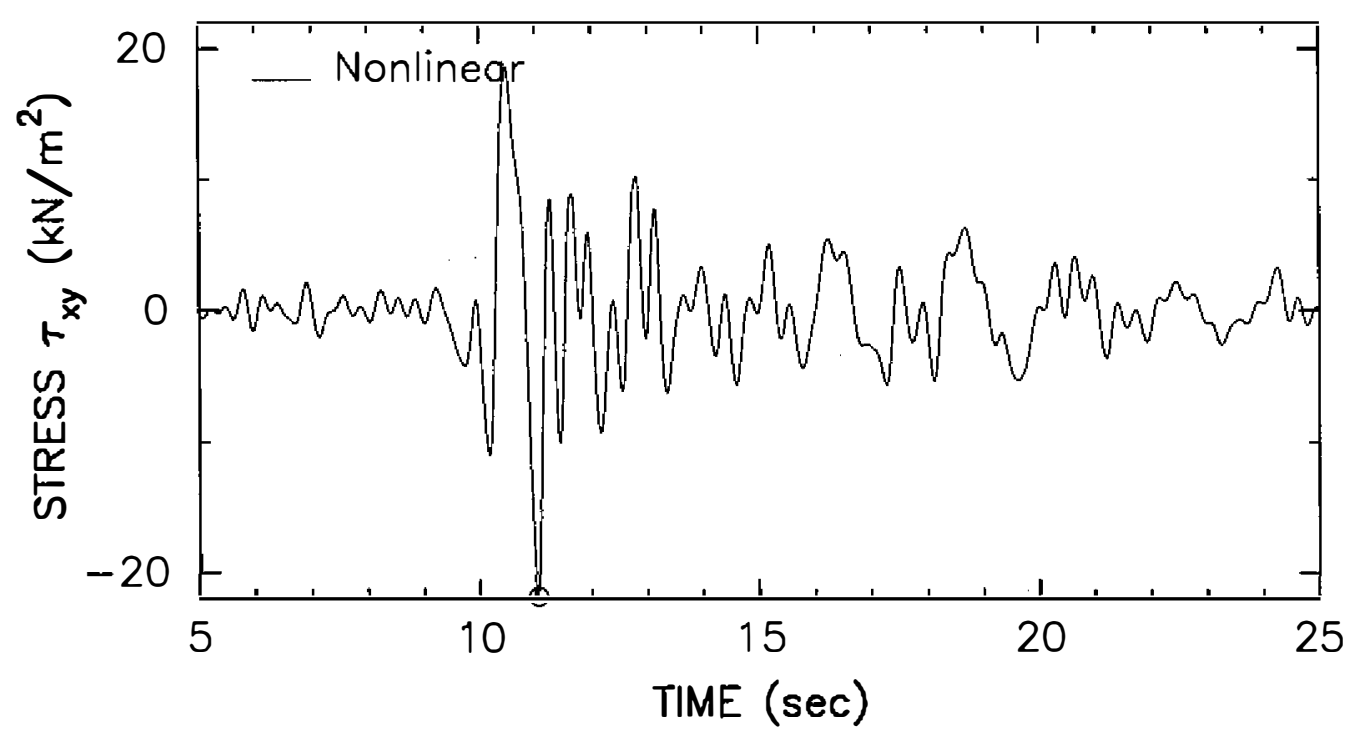

Fig. 11. The stress-strain relationship (a) and stress variation with time (b) at the $10 \mathrm{~m}$ depth of the Lotung downhole site in the 20 May 1986 mainshock event (No. 7). 
depths. However, this linear model does not work well for the strong motion recordings. In order to solve the problem, the nonlinear numerical scheme (DESRA-2) is used and can improve the simulation results. Basically, the synthetic result is consistent with the observed data in amplitude and phase although the PGA value at surface is still underestimated. Overall, the nonlinear simulation is feasible to predict the strong motions for a horizontal layered structure.

Acknowledgments We would like to express our thanks to Professor C.Y. Wang and the anonymous reviewer for their valuable suggestions. This research was supported by the National Science Council under grant number NSC 89-2116-M-194-003. The Institute of Earth Sciences, Academia Sinica supplied the strong-motion data. The support of these organizations is gratefully acknowledged.

\section{REFERENCES}

Aki, K., and K. Irikura, 1991: Characterization and mapping of earthquake shaking for seismic zonation, Proc. 4th Intl. Conf. On Seismic Zonation. Vol. 1, 61-110.

Aguirre, J., K. Irikura, and K. Kudo, 1994: Estimation of Strong ground motions on hard rock and soft sediment sites in the Ashigara Valley using the empirical Green's function method. Bull. Disas. Prev. Res. Inst., Kyoto Univ., 44, 45-68.

Aguirre, J., and K. Irikura, 1995: Preliminary analysis of nonlinear site effects at Port Island vertical array station during the 1995 Hyogo-ken Nanbu Earthquake. J. Natural Disaster Science, 16, 49-58.

Aguirre, J., and K. Irikura, 1997: Nonlinearity, liquefaction, and velocity variation of soft soil layers in Port Island, Kobe, during the Hyogoken-Nanbu Earthquake. Bull. Seism. Soc. Am., 87, 1244-1258.

Anderson, D. G., and Y. K. Tang, 1989: Summary of soil characterization program for the Lotung large-scale seismic experiment, Proc. EPRI/NRC/TPC workshop on seismic soil - structure interaction analysis technique using data from Lotung, Taiwan, Electric Power Research Institute, Palo Alto, CA, Vol. 1, 4-1 - 4-20.

Beresnev, I. A., K. L. Wen, and Y. T. Yeh, 1995a: Nonlinear soil amplification: its corroboration in Taiwan. Bull. Seism. Soc. Am., 85, 496-515.

Beresnev, I. A., K. L. Wen, and Y. T. Yeh, 1995b: Seismological evidence for nonlinear elastic ground behavior during large earthquakes. Soil Dyn. Earthquake Eng., 14, 103114.

Beresnev, I. A., G. M. Atkinson, P. A. Johnson, and E. H. Field, 1998a: Stochastic finite-fault modeling of ground motions from the 1994 Northridge, California, earthquake. II. widespread nonlinear response at soil sites. Bull. Seism. Soc. Am., 88, 1402-1410.

Beresnev, I. A., E. H. Field, K. V. D. Abeele, and P. A. Johnson, 1998b: Magnitude of nonlinear sediment response in Los Angeles Basin during the 1994 Northridge, California, earthquake. Bull. Seism. Soc. Am., 88, 1079-1084.

Borja, R. I., and W. H. Wu, 1994: Vibration of foundations on incompressible soils with no elastic region. J. Geotech. Engrg., ASCE, 120, 1570-1592.

Borja, R. I., H. Y. Chao, F. J. Montans, and C. H. Lin, 1999: Nonlinear ground response at 
Lotung LSST site. J. Geotechnical and Geoenvironmental Engineering, 125, 187-197.

Borja, R. I., C. H. Lin, K. M. Sama, and G. M. Masada, 2000: Modelling non-linear ground response of non-liquefiable soils. Earththquake Eng. Struct. Dyn,. 29, 63-83.

Chin, B. H., and K. Aki, 1991: Simultaneous study of the source, path, and site effects on strong ground motion during the 1989 Loma Prieta earthquake: a preliminary result on pervasive nonlinear site effects. Bull. Seism. Soc. Am., 81, 1859-1884.

Cultrera G., D. M. Boore, W. B. Joyner, and C. M. Dietel, 1999: Nonlinear soil response in the vicinity of the Van Norman Complex following the 1994 Northridge, California, earthquake. Bull. Seism. Soc. Am., 89, 1214-12321.

Haskell, N. A., 1953: The dispersion of surface waves on multilayered media. Bull. Seism. Soc. Am., 43, 17-35.

Haskell, N. A., 1960: Crustal reflection of plane SH waves. J. Geophys. Res., 65, 4147-4150.

Haskell, N. A., 1962: Crustal reflection of plane P and SV waves. J. Geophys. Res., 67, 47514767.

Hartzell, S., 1998: Variability in nonlinear sediment response during the 1994 Northridge, California, earthquake. Bull. Seism. Soc. Am., 88, 1426-1437.

HCK, 1986: 'Geophysical Survey Report of Lo-Tung Project for Taiwan Power Company', Technical Report, HCK Geophysical Company.

Huang, H. C., 1994: Canyon-topography and layered-structure effects on ground motion, Ph.D. Thesis, National Central University, Taiwan, 170pp.

Huang, H.C., and H. C. Chiu, 1996: Estimation of site amplification from Dahan downhole recordings. Earthquake Eng. Struct. Dyn., 25, 319-332.

Joyner, W. B., and A. T. F. Chen, 1975: Calculation of nonlinear ground response in earthquakes. Bull. Seism. Soc. Am., 65, 1315-1336.

Lee, M. K. W., and W. D. L. Finn, 1982: Dynamic effect stress response analysis of soil deposits with energy transmitting boundary including assessment of liquefaction potential, Rev., Dept. of Civil Eng., Soil Mechanics Series No. 38, the Univ. of British Columbia, Vancouver, Canada.

Masing, G., 1926: Eigenspannungen und Verfestigung beim Messing, in Proc. 2nd Int. Congress of Applied Mech., 323-335.

Ni, S. D., R. V. Siddharthan, and J. G. Anderson, 1997: Characteristics of nonlinear response of deep saturated soil deposits. Bull. Seism. Soc. Am., 87, 342-355.

Ordas, M., and E. Faccioli, 1994: Site response analysis in the valley of Mexico: selection of input motion and extent of nonlinear soil behavior. Earthquake Eng. Struct. Dyn., 23, 895-908.

Satoh, T., T. Sato, and H. Kawase, 1995: Nonlinear behavior of soil sediments identified by using borehole records observed at Ashigara Valley, Japan. Bull. Seism. Soc. Am., 85, 1821-1834.

Schnabe1, P. B., J. Lysmer, and H. B. Seed, 1972: SHAKE: a computer program for earthquake response analysis of horizontally layered sites, Report EERC 72-12, Earthquake Engineering Research Center, University of California, Berkeley.

Seed, H. B., and I. M. Idriss, 1970: Soil Moduli and damping factors for dynamic response 
analysis, Report EERC 70-10, Earthquake Engineering Research Centre, University of California, Berkeley.

Streeter, V. L., E. B. Wylie, and F. E. Richart, 1974: Soil motion computation by charachteristic method. J. Geotech. Eng. Div. ASCE, 100, 247-263.

$\mathrm{Su}, \mathrm{F}$., J. G. Anderson, and Y. Zeng, 1998: Study of weak and strong ground motion including nonlinearity from the Northridge, California, earthquake sequence. Bull. Seism. Soc. Am., 88, 1411-1425.

Wen, K. L., 1994: Nonlinear soil response in ground motion. Earthquake Eng. Struct. Dyn., 23, 599-608.

Wen, K. L., and Y. T. Yeh, 1984: Seismic velocity structure beneath the SMART1 array. Bull. Inst. Earth Sci.. Acad. Sin., 4, 51-72.

Wen, K. L., I. A. Beresnev, and Y. T. Yeh, 1994: Nonlinear soil amplification inferred from downhole strong seismic motion data. Geophys. Res. Lett., 21, 2625-2628.

Wen, K. L., I. A. Beresnev, and Y. T. Yeh, 1995: Investigation of nonlinear site amplification at two downhole strong ground motion arrays in Taiwan. Earthquake Eng. Struct. Dyn., 24, 313-324.

Yu, G., J. G. Anderson, and R. Siddharthan, 1993: On the characteristics of nonlinear soil response. Bull. Seism. Soc. Am., 83, 218-244. 